\title{
URS AND URSIMS FOR P-ADIC MEROMORPHIC FUNCTIONS INSIDE A DISC
}

\author{
ABDELBAKI BOUTABAA AND ALAIN ESCASSUT \\ Laboratoire de Mathématiques Pures, Université Blaise Pascal (Clermont-Ferrand), \\ Les Cézeaux, 63177 Aubiere Cedex, France (boutabaa@ucfma.univ-bpclermont.fr; \\ escassut@ucfma.univ-bpclermont.fr)
}

(Received 18 June 1999)

\begin{abstract}
Let $K$ be an algebraically closed field of characteristic zero, complete for an ultrametric absolute value. We show that the $p$-adic main Nevanlinna Theorem holds for meromorphic functions inside an 'open' disc in $K$. Let $P_{n, c}$ be the Frank-Reinders's polynomial

$$
(n-1)(n-2) X^{n}-2 n(n-2) X^{n-1}+n(n-1) X^{n-2}-c \quad(c \neq 0, c \neq 1, c \neq 2)
$$

and let $S_{n, c}$ be the set of its $n$ distinct zeros. For every $n \geqslant 7$, we show that $S_{n, c}$ is an $n$-points unique range set (counting multiplicities) for unbounded analytic functions inside an 'open disc', and for every $n \geqslant 10$, we show that $S_{n, c}$ is an $n$-points unique range set ignoring multiplicities for the same set of functions. Similar results are obtained for meromorphic functions whose characteristic function is unbounded: we obtain unique range sets ignoring multiplicities of 17 points. A better result is obtained for an analytic or a meromorphic function $f$ when its derivative is 'small' comparatively to $f$. In particular, for every $n \geqslant 5$ we show that $S_{n, c}$ is an $n$-points unique range set ignoring multiplicities for unbounded analytic functions with small derivative. Actually, in each case, results also apply to pairs of analytic functions when just one of them is supposed unbounded. The method we use is based upon the $p$-adic Nevanlinna Theory, and Frank-Reinders's and Fujimoto's methods used for meromorphic functions in $\mathbb{C}$. Among other results, we show that the set of functions having a bounded characteristic function is just the field of fractions of the ring of bounded analytic functions in the disc.
\end{abstract}

Keywords: meromorphic; Nevanlinna; ultrametric; urs; ursim

AMS 2000 Mathematics subject classification: Primary 12H25

Secondary 12J25; 46S10

\section{Introduction and theorems}

\section{Definitions and notation}

We denote by $K$ an algebraically closed field of characteristic zero, complete for an ultrametric absolute value and by $\mathbb{P}^{1}(K)$ the one-dimensional projective space over $K$.

We denote by $\mathcal{A}(K)$ the set of entire functions in $K$, and by $\mathcal{M}(K)$ the set of meromorphic functions in $K$, i.e. the field of fractions of $\mathcal{A}(K)$. Given $a \in K$ and $r>0$, $d(a, r)$ is the $\operatorname{disc}\{x \in K|| x-a \mid \leqslant r\}$ and $d\left(a, r^{-}\right)$is the $\operatorname{disc}\{x \in K|| x-a \mid<r\}$. In the same way, we denote by $\mathcal{A}\left(d\left(a, r^{-}\right)\right)$the set of analytic functions in $d\left(a, r^{-}\right)$, i.e. the 
$K$-algebra of power series $\sum_{n=0}^{\infty} a_{n}(x-a)^{n}$ converging in $d\left(a, r^{-}\right)$, and by $\mathcal{M}\left(d\left(a, r^{-}\right)\right)$ the set of meromorphic functions inside $d\left(a, r^{-}\right)$, i.e. the field of fractions of $\mathcal{A}\left(d\left(a, r^{-}\right)\right)$.

In the sequel, $J$ will denote an interval of the form $[\rho, R[$, with $\rho>0$.

Let $\log$ be the real logarithm function of base $p>1$. Let $R \in] 0,+\infty[$ and let $f \in$ $\mathcal{M}\left(d\left(0, R^{-}\right)\right)$such that 0 is neither a zero nor a pole of $f$. Let $\left.r \in\right] \rho, R[$. We will denote by $Z(r, f)$ the counting function of zeros of $f$ in $d(0, R) \backslash\{0\}$, i.e. if $\left(a_{n}\right)$ is the finite or infinite sequence of zeros of $f$ in $d\left(0, R^{-}\right) \backslash\{0\}$, with respective multiplicity order $s_{n}$, we put

$$
Z(r, f)=\sum_{\left|a_{n}\right| \leqslant r} s_{n}\left(\log r-\log \left|a_{n}\right|\right) \quad \text { and } \quad \bar{Z}(r, f)=\sum_{\left|a_{n}\right| \leqslant r}\left(\log r-\log \left|a_{n}\right|\right) .
$$

In the same way, considering the sequence $\left(b_{n}\right)$ of poles of $f$ in $d(0, r) \backslash\{0\}$, with respective multiplicity order $t_{n}$, we put

$$
N(r, f)=\sum_{\left|b_{n}\right| \leqslant r} t_{n}\left(\log r-\log \left|b_{n}\right|\right) \quad \text { and } \quad \bar{N}(r, f)=\sum_{\left|b_{n}\right| \leqslant r}\left(\log r-\log \left|b_{n}\right|\right) .
$$

Here, we need to generalize these counting functions to functions having a pole or a zero at 0 . So, if $f$ admits a zero (respectively, a pole) of order $q$ at 0 , we put $h=x^{-q} f$ (respectively, $h=x^{q} f$ ) and $Z(r, f)=Z(r, h), N(r, f)=N(r, h)$.

The Nevanlinna function $T(r, f)$ is defined, when $f$ has neither zeros nor poles at 0 , by

$$
T(r, f)=\max (Z(r, f)+\log |f(0)|, N(r, f)) .
$$

And if $f$ has either a zero or a pole at 0 , we put

$$
T(r, f)=\max (Z(r, h), N(r, h)) .
$$

Finally, if $S$ is a subset of $K$ we will denote by $Z_{0}^{S}\left(r, f^{\prime}\right)$ the counting function of zeros of $f^{\prime}$, excluding 0 and those which are zeros of $f-a$ for any $a \in S$.

In [16] Lazard considered this problem: given a sequence $\left(a_{n}\right)$ in $d\left(0, R^{-}\right)$and a sequence of positive integers $\left(q_{n}\right)$, can we find $f \in \mathcal{A}\left(d\left(0, R^{-}\right)\right)$admitting each $a_{n}$ as a zero of order $q_{n}$, and having no other zero? In the general case, he showed the answer is no, except if $K$ is supposed to be spherically complete. Here this problem has several consequences. However, we can deal with the problem thanks to Lemma 2.4, which recalls Theorem 25.5 in [10], so that we could find a function $\phi$ (respectively, $\psi)$ in $\mathcal{A}\left(d\left(a, R^{-}\right)\right.$) whose counting function of zeros isn't much bigger than the counting function of zeros (respectively, poles) of $f$.

The $p$-adic Nevanlinna main theorem for meromorphic functions in all $K$ was proven in $[\mathbf{2}, \mathbf{3}]$. In $[\mathbf{1 5}]$, another statement was made concerning meromorphic functions inside the disc $d\left(0,1^{-}\right)$. However, its proof did not take into account the fact that, as explained above, given $f \in \mathcal{M}\left(d\left(a, R^{-}\right)\right)$, we cannot be sure to find $\phi, \psi \in \mathcal{A}\left(d\left(a, R^{-}\right)\right)$such that the zeros of $\phi$ (respectively, of $\psi$ ) be exactly the zeros (respectively, the poles) of $f$ 
counting multiplicities, which put in doubt the claim in [15]. Here, we shall be able to give a complete proof of the second main Nevanlinna theorem inside any disc $d\left(0, R^{-}\right)$ (Theorems 1.4 and $1.4^{\prime}$ ).

Notation. For all $r \in] 0, R\left[\right.$ we denote by $\|\cdot\|_{a, r}$ the norm of uniform convergence in the disc $d(a, r)$, i.e. given $f(x)=\sum_{j=0}^{\infty} a_{j} x^{j}$, then $\|f\|_{a, r}=\sup \{|f(x)| \mid x \in d(a, r)\}=$ $\sup _{n \in \mathbb{N}}\left|a_{n}\right| r^{n}$.

We will denote by $\mathcal{A}_{b}\left(d\left(a, R^{-}\right)\right)$the $K$-subalgebra of $\mathcal{A}\left(d\left(a, R^{-}\right)\right)$consisting of the bounded analytic functions $f \in \mathcal{A}\left(d\left(a, R^{-}\right)\right)$, and by $\mathcal{M}_{b}\left(d\left(a, R^{-}\right)\right)$the field of fractions of $\mathcal{A}_{b}\left(d\left(a, R^{-}\right)\right)$. Then, we will denote by $\mathcal{A}_{u}\left(d\left(a, R^{-}\right)\right)$the set $\mathcal{A}\left(d\left(a, R^{-}\right)\right) \backslash \mathcal{A}_{b}\left(d\left(a, R^{-}\right)\right)$, and similarly, we put $\mathcal{M}_{u}\left(d\left(a, R^{-}\right)\right)=\mathcal{M}\left(d\left(a, R^{-}\right)\right) \backslash \mathcal{M}_{b}\left(d\left(a, R^{-}\right)\right)$.

In the same way, we will denote by $\mathcal{A}_{b d}\left(d\left(a, R^{-}\right)\right)$the $K$-subvector space of $\mathcal{A}\left(d\left(a, R^{-}\right)\right)$ consisting of the $f \in \mathcal{A}\left(d\left(a, R^{-}\right)\right)$such that $f^{\prime} \in \mathcal{A}_{b}\left(d\left(a, R^{-}\right)\right)$.

Let $\alpha \in] 0,1\left[\right.$. We will denote by $\mathcal{M}_{\alpha}\left(d\left(a, R^{-}\right)\right)$the set of $f \in \mathcal{M}_{u}\left(d\left(a, R^{-}\right)\right)$such that $\lim \sup _{r \rightarrow R} Z\left(r, f^{\prime}(a+x)\right) / T(r, f(a+x))<\alpha$. Finally, we will denote by $\mathcal{A}_{\alpha}\left(d\left(a, R^{-}\right)\right)$the subset $\mathcal{A}\left(d\left(a, R^{-}\right)\right) \cap \mathcal{M}_{\alpha}\left(d\left(a, R^{-}\right)\right)$of $\mathcal{A}_{u}\left(d\left(a, R^{-}\right)\right)$. Then it is easily checked that the set $\mathcal{M}_{\alpha}\left(d\left(a, R^{-}\right)\right)$contains at least all functions of the form $h \circ f$, with $f \in \mathcal{A}_{\alpha}\left(d\left(a, R^{-}\right)\right)$, and $h$ a linear fractional function.

\section{Remarks.}

(1) As recalled in Lemma 2.1 below, given $f \in \mathcal{A}\left(d\left(a, R^{-}\right)\right)$the functions $T(r, f)$, $Z(r, f),-\log \left(\|f\|_{a, r}\right)$ are equivalent up to an additive constant. Consequently, a function $f \in \mathcal{A}\left(d\left(a, R^{-}\right)\right)$belongs to $\mathcal{A}_{\alpha}\left(d\left(a, R^{-}\right)\right)$if and only if

$$
\limsup _{r \rightarrow R}\left(\frac{\log \left(\left\|f^{\prime}\right\|_{a, r}\right)}{\log \left(\|f\|_{a, r}\right)}\right)<\alpha .
$$

(2) If $f \in \mathcal{A}_{u}\left(d\left(a, R^{-}\right)\right)$has a bounded derivative, it belongs to $\mathcal{A}_{\alpha}\left(d\left(a, R^{-}\right)\right)$. For example, this is the case of $h(x)=\log (1+x)=\sum_{n=0}^{\infty}(-1)^{n} x^{n+1} /(n+1)$ in $\mathcal{A}\left(d\left(0,1^{-}\right)\right)$.

(3) Now, we place ourselves in $\mathbb{C}_{p}$ and consider $g(x)=\sum_{n=2}^{\infty} x^{p^{n}} / p^{2 n} \in \mathcal{A}\left(d\left(0,1^{-}\right)\right)$: its derivative is not bounded but $g$ belongs to $\mathcal{A}_{\alpha}\left(d\left(0,1^{-}\right)\right)$for all $\left.\alpha \in\right] 0,1[$.

Theorem 1.1 is not completely obvious and again requires the use of Lemma 2.4. It also answers a question asked by $\mathrm{Hu}$ and Yang [22]. An analogous statement was made in $[\mathbf{1 5}]$, but for the same reason as for Theorem 1.4, its proof was not complete.

Theorem 1.1. Let $f \in \mathcal{M}\left(d\left(0, R^{-}\right)\right)$. Then $f$ belongs to $\mathcal{M}_{b}\left(d\left(0, R^{-}\right)\right)$if and only if $T(r, f)$ is bounded in $J$.

With the help of Theorem 1.1 we will prove Theorem 1.2.

Theorem 1.2. Let $\omega, \theta, \chi, \tau \in \mathcal{M}_{b}\left(d\left(a, R^{-}\right)\right)$be such that $\omega \tau-\chi \theta$ is not identically zero and let $h(u)=(\omega u+\theta) /(\chi u+\tau)$. For every $f \in \mathcal{M}\left(d\left(a, R^{-}\right)\right)$such that $\chi f+\tau$ is not identically zero, $h(f)$ belongs to $\mathcal{M}_{b}\left(d\left(a, R^{-}\right)\right.$) (respectively, $\mathcal{M}_{u}\left(d\left(a, R^{-}\right)\right)$) if and only if so does $f$. Moreover, we have $T(r, f)=T(r, h(f))+O(1)$. 
Corollary 1.3. Let $f \in \mathcal{M}_{u}\left(d\left(a, R^{-}\right)\right)$, and let $h \in \mathcal{M}_{b}\left(d\left(a, R^{-}\right)\right), h \neq 0$. Then $f h$ belongs to $\mathcal{M}_{u}\left(d\left(a, R^{-}\right)\right)$.

Remarks.

(4) If $f, g \in \mathcal{M}_{u}\left(d\left(0, R^{-}\right)\right)$satisfy $T(r, f)=T(r, g)+O(1)$, this does not imply that $g$ is of the form $(\omega f+\theta) /(\chi f+\tau)$, with $\omega, \theta, \chi, \tau \in \mathcal{M}_{b}\left(d\left(0, R^{-}\right)\right)$. For example, let $h \in \mathcal{A}_{u}\left(d a, r^{-}\right)$, and consider $f=h^{2}$ and $g=h^{2}+h$. Clearly, $g$ is not a linear fractional function in $f$.

Theorem 1.4. Let $\alpha_{1}, \ldots, \alpha_{q} \in K$, with $q \geqslant 2$, let $S=\left\{\alpha_{1}, \ldots, \alpha_{q}\right\}$, and let $f \in$ $\mathcal{M}\left(d\left(0, R^{-}\right)\right)$. There exists $C \in \mathbb{R}$ such that

$$
(q-1) T(r, f) \leqslant \sum_{j=1}^{q} \bar{Z}\left(r, f-\alpha_{j}\right)+Z\left(r, f^{\prime}\right)-Z_{0}^{S}\left(r, f^{\prime}\right)+C \quad \forall r \in J .
$$

Moreover, if $f$ belongs to $\mathcal{A}\left(d\left(0, R^{-}\right)\right)$, then

$$
q T(r, f) \leqslant \sum_{j=1}^{q} \bar{Z}\left(r, f-\alpha_{j}\right)+Z\left(r, f^{\prime}\right)-Z_{0}^{S}\left(r, f^{\prime}\right)+C \quad \forall r \in J .
$$

Theorem 1.4'. Let $\alpha_{1}, \ldots, \alpha_{q} \in K$, with $q \geqslant 2$, let $S=\left\{\alpha_{1}, \ldots, \alpha_{q}\right\}$, and let $f \in$ $\mathcal{M}\left(d\left(0, R^{-}\right)\right)$. There exists $C \in \mathbb{R}$ such that

$$
(q-1) T(r, f) \leqslant \sum_{j=1}^{q} \bar{Z}\left(r, f-\alpha_{j}\right)+\bar{N}(r, f)-Z_{0}^{S}\left(r, f^{\prime}\right)+C \quad \forall r \in J .
$$

Definition 1.5. Given a family of functions $\mathcal{F}$ defined in $d\left(a, R^{-}\right)$, with values in $\mathbb{P}^{1}(K)$, a set $S$ in $K$ is called an ursim for $\mathcal{F}$ if for any non-constant functions $f, g \in$ $\mathcal{F}$ satisfying $f^{-1}(S)=g^{-1}(S)$, these functions are equal. It is known that the field of complex meromorphic functions admits ursims of 17 points, and the algebra of complex entire functions admits ursims of 10 points. In [11] as in [6] $n$-points ursims for $\mathcal{A}(K)$ are constructed for every $n \geqslant 9$, and in $[\mathbf{6}]$ we constructed $n$-points ursims for $\mathcal{M}(K)$ for every $n \geqslant 16$.

For a subset $S$ of $K$ and $f \in \mathcal{M}\left(d\left(a, R^{-}\right)\right)$we denote by $E(f, S)$ the set in $\left(d\left(a, R^{-}\right)\right) \times$ $\mathbb{N}^{*}:$

$$
\bigcup_{a \in S}\left\{(z, q) \in\left(d\left(a, R^{-}\right)\right) \times \mathbb{N}^{*} \mid z \text { a zero of order } q \text { of } f(x)-a\right\} .
$$

Let $\mathcal{F}$ be a non-empty subset of $\mathcal{M}\left(d\left(a, R^{-}\right)\right)$. A subset $S$ of $K$ is called a unique range set (an urs in brief) for $\mathcal{F}$ if for any non-constant $f, g \in \mathcal{F}$ such that $E(f, S)=E(g, S)$, one has $f=g$.

It is known that the algebra of complex entire functions admits urs of 7 points, and that the field of complex meromorphic functions admits urs of 11 points [12].

In [4] we showed that $\mathcal{A}(K)$ admits urs of 3 points. More generally, in [9] it was proven that urs for $\mathcal{A}(K)$ are the urs for polynomials (characterized in [4], as the sets 
which are not preserved by any affine transformation, but the identity). According to the remarkable method due to Franck and Reinders in [12], it is known that $\mathcal{M}(K)$ admits urs of 10 points.

Many other papers were published about properties of uniqueness or strong relationship, for functions sharing a certain finite set of values, counting or not multiplicities $[\mathbf{1}, \mathbf{7}, \mathbf{8}, \mathbf{1 3}, \mathbf{1 4}, \mathbf{1 6}-\mathbf{2 1}]$.

Here, we consider problems of uniqueness for unbounded functions in $\mathcal{A}\left(d\left(a, R^{-}\right)\right)$, and for similar meromorphic functions. We will use a process using the $p$-adic Nevanlinna Theory, and based upon Frank-Reinders's method in [12] for certain cases, and upon Fujimoto's method [13] (which itself has a relationship with the method of FrankReinders) for the most general case.

Notation. For all the sequel, we choose $c \in K \backslash\{0,1,2\}, n \in \mathbb{N}$ and denote by $P_{n, c}$ the polynomial $(n-1)(n-2) X^{n}-2 n(n-2) X^{n-1}+n(n-1) X^{n-2}-c$ and by $S(n, c)$ the set of zeros of $P_{n, c}$. Since $K$ has characteristic zero, and since $c \neq 0$ and 2 , it is seen that all zeros of $P$ are simple.

Theorem 1.6. Let $f \in \mathcal{M}_{u}\left(d\left(a, R^{-}\right)\right)$and let $g \in \mathcal{M}\left(d\left(a, R^{-}\right)\right)$be two different nonconstant functions satisfying $f^{-1}(S(n, c))=g^{-1}(S(n, c))$. Then $n \leqslant 16$. Moreover, if $f \in$ $\mathcal{A}_{u}\left(d\left(a, R^{-}\right)\right)$and if $g \in \mathcal{A}\left(d\left(a, R^{-}\right)\right)$, then $n \leqslant 9$. Further, if $f, g \in \mathcal{M}_{1 / 5}\left(d\left(a, R^{-}\right)\right)$, then $n \leqslant 7$. Further, if $f, g \in \mathcal{A}_{1 / 5}\left(d\left(a, R^{-}\right)\right)$, or if $f \in \mathcal{A}_{1 / 5}\left(d\left(a, R^{-}\right)\right)$and $g \in \mathcal{A}_{b d}\left(d\left(a, R^{-}\right)\right)$, then $n \leqslant 4$.

Corollary 1.7. Let $n \geqslant 17$. Then $S(n, c)$ is an ursim for $\mathcal{M}_{u}\left(d\left(a, R^{-}\right)\right)$.

Corollary 1.8. Let $n \geqslant 10$. Then $S(n, c)$ is an ursim for $\mathcal{A}_{u}\left(d\left(a, R^{-}\right)\right)$.

Corollary 1.9. Let $n \geqslant 8$. Then $S(n, c)$ is an ursim for $\mathcal{M}_{1 / 5}\left(d\left(a, R^{-}\right)\right)$.

Corollary 1.10. Let $n \geqslant 5$. Then $S(n, c)$ is an ursim for $\mathcal{A}_{1 / 5}\left(d\left(a, R^{-}\right)\right)$.

\section{Examples.}

(1) As noticed above, the $p$-adic logarithm function $\log (1+x)$ defined in $d\left(0,1^{-}\right)$lies in $\mathcal{A}_{b d}\left(d\left(0,1^{-}\right)\right)$. So, given $n \geqslant 5$ and $f \in \mathcal{A}_{1 / 5}\left(d\left(0,1^{-}\right)\right)$such that $f^{-1}(S(n, c))=$ $\log ^{-1}(S(n, c))$, then $f(x)$ is identically equal to $\log (1+x)$.

(2) Consider again the function $g(x)=\sum_{n=2}^{\infty} x^{p^{n}} / p^{2 n} \in \mathcal{A}\left(d\left(0,1^{-}\right)\right)$defined above, and let $f \in \mathcal{A}_{1 / 5}\left(d\left(0,1^{-}\right)\right)$be such that $f^{-1}(S(n, c))=g^{-1}(S(n, c))$, with $n \geqslant 5$. Since $g$ belongs to $\mathcal{A}_{1 / 5}\left(d\left(0,1^{-}\right)\right)$, we have $f=g$.

Theorem 1.11. Let $f, g \in \mathcal{M}_{u}\left(d\left(a, R^{-}\right)\right)$be two different non-constant functions satisfying $E(f, S(n, c))=E(g, S(n, c))$. Then $n \leqslant 10$. Moreover, if $f, g \in \mathcal{A}_{u}\left(d\left(a, R^{-}\right)\right)$, then $n \leqslant 6$.

Corollary 1.12. Let $n \geqslant 11$. Then $S(n, c)$ is an urs for $\mathcal{M}_{u}\left(d\left(a, R^{-}\right)\right)$.

Corollary 1.13. Let $n \geqslant 7$. Then $S(n, c)$ is an urs for $\mathcal{A}_{u}\left(d\left(a, R^{-}\right)\right)$. 
We cannot obtain similar results on bounded functions. However, in a very particular case we obtain a set of uniqueness $T$ which is $\log ^{-1}(S(n, c))$, and thereby is infinite.

Application to bounded functions. Let $n \geqslant 10$, let $T=\log ^{-1}(S(n, c))$, let $f, g \in$ $\mathcal{A}\left(d\left(a, R^{-}\right)\right)$be such that $f\left(d\left(a, R^{-}\right)\right)=g\left(d\left(a, R^{-}\right)\right)=d\left(1,1^{-}\right)$, and $f^{-1}(T)=g^{-1}(T)$. Then $f=g$.

Proof. Indeed, as $f\left(d\left(a, R^{-}\right)\right)=g\left(d\left(a, R^{-}\right)\right)=d\left(1,1^{-}\right)$we can define both Log $\circ f$, $\log \circ g$ and check that both are unbounded in $d\left(a, R^{-}\right)$. Therefore, we can apply Corollary 1.7 to them. But the hypothesis $f^{-1}(T)=g^{-1}(T)$ just means that $(\log \circ f)^{-1}(S(n, c))=$ $(\log \circ g)^{-1}(S(n, c))$.

\section{Remarks.}

(5) In [11] $n$-points ursim for $\mathcal{A}(K)$ are obtained whenever $n \geqslant 9$, and $n$-points ursim for $\mathcal{M}(K)$ are obtained whenever $n \geqslant 16$. The method used in this previous work is close to this one and could also let us obtain the same results as those we have obtained here, as far as $\mathcal{A}\left(d\left(a, R^{-}\right)\right)$and $\mathcal{M}\left(d\left(a, R^{-}\right)\right)$are concerned. However, in [11], with $\mathcal{A}(K)$ or $\mathcal{M}(K)$ we had the benefit of one point from the intervention of a term $-\log r$ which is not efficient when $r$ is bounded. On the other hand, Fujimoto's method here is more efficient when $\mathcal{A}_{\alpha}(K)$ or $\mathcal{M}_{\alpha}(K)$ are concerned.

(6) In [4], we had $n$-points urs for $\mathcal{A}(K)$ whenever $n \geqslant 3$. But the method used was completely different, avoiding the Nevanlinna Theory, and mainly using the fact that meromorphic functions having neither any zeros nor any poles are just constants. The claim in [4] is generalized in [9], showing that all urs for $\mathcal{A}(K)$ are urs for $K[x]$, i.e. the urs for $\mathcal{A}(K)$ are the sets which are never preserved by affine mappings other than the identity. But the method is based upon properties of algebraic curves that we cannot reproduce here in $\mathcal{A}\left(d\left(a, R^{-}\right)\right)$.

\section{The proofs}

Notation. In $K$, the valuation $v$ is defined as $v(x)=-\log |x|$.

Given $f(x)=\sum_{n=0}^{\infty} a_{n} x^{n} \in \mathcal{A}(K)$ and $\mu \in \mathbb{R}$, we denote by $v(f, \mu)$ the valuation function defined by $v(f, \mu)=\inf _{n \in \mathbb{N}} v\left(a_{n}\right)+n \mu$. This valuation can be continued to $\mathcal{M}(K)$ by setting $v(f / g, \mu)=v(f, \mu)-v(g, \mu)$.

Let $\alpha \in K$ and $h \in \mathcal{M}(K)$. If $h$ has a zero (respectively, a pole) of order $q$ at $\alpha$, we put $\omega_{\alpha}(h)=q$ (respectively, $\left.\omega_{\alpha}(h)=-q\right)$. If $h(\alpha) \neq 0$ and $\infty$, we put $\omega_{\alpha}(h)=0$.

Both Lemmas 2.1 and 2.2 are classical. Lemma 2.1 is a consequence of Theorem 23.13 in [10], and Lemma 2.2 is immediate.

Lemma 2.1. Let $f \in \mathcal{M}\left(d\left(0, R^{-}\right)\right)$be such that 0 is neither a zero nor a pole of $f$. Then $v(f,-\log r)+Z(r, f)-N(r, f)$ is bounded in $J$. Moreover, $v(f,-\log r)=v(f(0))-$ $Z(r, f)+N(r, f) \forall r \in] 0, R[$. 
Lemma 2.2. Let $f \in \mathcal{A}\left(d\left(0, R^{-}\right)\right)$let $\left(a_{n}\right)_{n \in L}$, be the finite or infinite sequence of its zeros, and for each $n \in L$, let $s_{n}$ be the multiplicity order of $a_{n}$. Let $g \in \mathcal{A}\left(d\left(0, R^{-}\right)\right)$ admitting each $a_{n}$ as a zero of order $u_{n} \geqslant s_{n}$. Then $g$ factorizes in $\mathcal{A}\left(d\left(0, R^{-}\right)\right)$in the form $f h$, with $h \in \mathcal{A}\left(d\left(0, R^{-}\right)\right)$.

Remark. Consider a sequence $\left(a_{n}\right)$ in $d\left(0, R^{-}\right)$such that $\lim _{n \rightarrow \infty}\left|a_{n}\right|=R$, and a sequence of integers $\left(q_{n}\right)$. As mentioned in the Introduction, we cannot ensure that there exists $f \in \mathcal{A}(d(0, r))$ admitting each $a_{n}$ as a zero of order $q_{n}$, exactly, and having no other zeros [16]. However, here we can deal with this problem, by using Theorem 25.5 in $[\mathbf{1 0}]$, that we translate in terms of valuation below. We just have to notice a misprint in the statement of [10, Theorem 25.5]: the function $f$ satisfying such properties belongs to $\mathcal{A}\left(d\left(0, r^{-}\right)\right)$, and not to $\mathcal{A}_{b}\left(d\left(0, r^{-}\right)\right)$: actually this is correctly explained in the introduction to Chapter 25, and the proof of Theorem 25.5 confirms this fact.

Theorem 2.3. Let $\left(a_{j}\right)_{j \in \mathbb{N}}$ be a sequence in $d\left(0, R^{-}\right) \backslash\{0\}$ such that $\left|a_{n}\right| \leqslant\left|a_{n+1}\right|$ for every $n \in \mathbb{N}$ and $\lim _{n \rightarrow \infty}\left|a_{n}\right|=R$. Let $\left(q_{n}\right)_{n \in \mathbb{N}}$ be a sequence in $\mathbb{N}^{*}$ and let $\varepsilon>0$. There exists $f \in \mathcal{A}\left(d\left(0, R^{-}\right)\right)$satisfying

(i) $f(0)=1$,

(ii) $-v\left(f, v\left(a_{n}\right)\right) \leqslant \sum_{j=0}^{n} q_{j} v\left(a_{j} / a_{n}\right)+\varepsilon$ whenever $n \in \mathbb{N}$,

(iii) for each $n \in \mathbb{N}, a_{n}$ is a zero of $f$ of order $z_{n} \geqslant q_{n}$.

Lemma 2.4. Let $f \in \mathcal{M}\left(d\left(0, R^{-}\right)\right)$, and let $\varepsilon>0$. There exist $\phi, \psi \in \mathcal{A}\left(d\left(0, R^{-}\right)\right)$ such that $f=\phi / \psi$, and $Z(r, \phi) \leqslant Z(r, f)+\varepsilon, Z(r, \psi) \leqslant N(r, f)+\varepsilon \forall r \in J$.

Proof. We can obviously assume $f(0)=1$. Next, the claim is obvious when $f$ has finitely many zeros and poles. So, we can assume that $f$ has infinitely many zeros or infinitely many poles. Let $\left(a_{n}\right)_{n \in \mathbb{N}}$ (respectively, $\left(b_{n}\right)_{n \in \mathbb{N}}$ ) be the sequence of zeros (respectively, poles) of $f$, and for each $n \in \mathbb{N}$, let $s_{n}$ (respectively, $t_{n}$ ) be the multiplicity order of $a_{n}$ (respectively, $\left.b_{n}\right)$. According to Theorem 2.3 there exists $\phi \in \mathcal{A}\left(d\left(0, R^{-}\right)\right)$satisfying $\phi(0)=1$, and admitting each $a_{n}$ as a zero of order $s_{n}^{\prime} \geqslant s_{n}$, such that $v(\phi,-\log r) \geqslant$ $\sum_{\left|a_{n}\right| \leqslant r} s_{j}\left(-\log r-v\left(a_{n}\right)\right)-\varepsilon \forall r \in J$, i.e.

$$
Z(r, \phi) \leqslant Z(r, f)+\varepsilon \quad \forall r \in J .
$$

Now, we put $\psi=\phi / f$. Then, by Lemma $2.2, \psi$ lies in $\mathcal{A}\left(d\left(0, R^{-}\right)\right)$, and by definition, each pole $b_{n}$ of $f$ is a zero of $\psi$ of multiplicity order $\geqslant t_{n}$. Moreover, we have $Z(r, \psi)=$ $N(r, f)+Z(r, \phi)-Z(r, f)$. Consequently, by $(2.1)$ we obtain $Z(r, \psi) \leqslant N(r, f)+\varepsilon$ and this ends the proof.

Proof of Theorem 1.1. We can obviously assume that $f(0) \neq 0, f(0) \neq \infty$. If $f \in$ $\mathcal{M}_{b}\left(d\left(0, R^{-}\right)\right)$, then $f$ is of the form $\phi / \psi$, with $\phi, \psi \in \mathcal{A}_{b}\left(d\left(0, R^{-}\right)\right)$. Consequently, both $Z(r, \phi), Z(r, \psi)$ are bounded, and therefore so are $Z(r, f)$ and $N(r, f)$ because obviously $Z(r, f) \leqslant Z(r, \phi), N(r, f) \leqslant Z(r, \psi)$, thereby $T(r, f)$ is bounded. Conversely, suppose that $T(r, f)$ is bounded. Then so are $Z(r, f)$ and $N(r, f)$. Now, by Lemma 2.4 we can find 
$\phi, \psi \in \mathcal{A}\left(d\left(0, R^{-}\right)\right)$such that $Z(r, \phi) \leqslant Z(r, f)+1, Z(r, \psi) \leqslant N(r, f)+1$, and $f=\phi / \psi$. Then by Lemma 2.1 both $v(\phi,-\log r), v(\phi,-\log r)$ are bounded when $r \in] 0,+\infty[$, and therefore so are $\|\phi\|_{0, r},\|\psi\|_{0, r}$, hence $f$ belongs to $\mathcal{M}_{b}\left(d\left(0, R^{-}\right)\right)$. This completes the proof.

Proof of Theorem 1.2. Let $g=h(f)$. By Lemma 2.4 we can write $f=\phi / \psi$ with $\phi, \psi \in \mathcal{A}\left(d\left(0, R^{-}\right)\right)$, and $Z(r, \phi) \leqslant Z(r, f)+1$, and $Z(r, \psi) \leqslant N(r, f)+1$. Without loss of generality, we can assume that $\omega, \theta, \chi, \tau$ lies in $\mathcal{A}_{b}\left(d\left(0, R^{-}\right)\right)$. Then we can see that

$$
\begin{aligned}
Z(r, g) & \leqslant Z(r, \omega \phi+\theta \psi)=-v(\omega \phi+\theta \psi,-\log r) \\
& \leqslant \max (-v(\omega,-\log r)-v(\phi,-\log r),-v(\theta,-\log r)-v(\psi,-\log r)) \\
& \leqslant T(r, f)+O(1),
\end{aligned}
$$

and similarly

$$
\begin{aligned}
N(r, g) & \leqslant Z(r, \chi \phi+\tau \psi)=-v(\chi \phi+\tau \psi,-\log r) \\
& \leqslant \max (-v(\chi,-\log r)-v(\phi,-\log r),-v(\tau,-\log r)-v(\psi,-\log r)) \\
& \leqslant T(r, f)+O(1) .
\end{aligned}
$$

Consequently, $T(r, g) \leqslant T(r, f)+O(1)$. But since $\omega \tau-\chi \theta$ is not identically zero, we can consider the inverse transformation in $\mathcal{M}\left(d\left(a, R^{-}\right)\right)$and we have $f=(\tau g-\theta) /(-\chi g+$ $\omega)$. Consequently, $T(r, f) \leqslant T(r, g)+O(1)$. So, in particular, $g$ lies in $\mathcal{M}_{b}\left(d\left(0, R^{-}\right)\right)$ (respectively, in $\mathcal{M}_{u}\left(d\left(0, R^{-}\right)\right)$) if and only if so does $f$.

Definition 2.5. We call a divisor a mapping $\psi$ from $d\left(0, R^{-}\right)$to $\mathbb{N}$ whose support is countable and has a finite intersection with each disc $d(a, r), \forall r<R$. The set of divisors is provided with a natural additive law. Moreover, if $\omega, \varphi$ are two divisors such that $\psi(\alpha) \geqslant \varphi(\alpha) \forall \alpha \in d\left(0, R^{-}\right)$, we can define the divisor $\psi-\varphi$.

Given $f \in \mathcal{M}(K)$, we can define the divisor $\mathcal{D}(f)$ as $\mathcal{D}(f)(\alpha)=0$ whenever $(\alpha) \neq 0$, and $\mathcal{D}(f)(\alpha)=s$ when $f$ has a zero of order $s$ at $\alpha$.

Finally, we denote by $\overline{\mathcal{D}}(f)$ the divisor defined as $\mathcal{D}(f)(\alpha)=0$ whenever $f(\alpha) \neq 0$, and $\mathcal{D}(f)(\alpha)=1$ when $f$ has a zero at $\alpha$.

Lemma 2.6. Let $f \in \mathcal{M}\left(d\left(0, R^{-}\right)\right)$. Then $Z\left(r, f^{\prime}\right) \leqslant Z(r, f)+\bar{N}(r, f)+O(1)(r \in J)$.

Proof. We can obviously assume that $f, f^{\prime}$ satisfy $f(0) \neq 0, f^{\prime}(0) \neq 0, f(0) \neq \infty$, $f^{\prime}(0) \neq \infty$.

Then, by Lemma 2.1, we have $Z(r, f)-N(r, f)=-v(f,-\log r)+v(f(0))$ and $Z\left(r, f^{\prime}\right)-$ $N\left(r, f^{\prime}\right)=-v\left(f^{\prime},-\log r\right)+v\left(f^{\prime}(0)\right)$. But $v\left(f^{\prime},-\log r\right) \geqslant v(f,-\log r)+\log r$, hence we obtain $Z\left(r, f^{\prime}\right) \leqslant N\left(r, f^{\prime}\right)-N(r, f)+Z(r, f)+O(1)$. But actually, $N\left(r, f^{\prime}\right)-N(r, f)=$ $\bar{N}(r, f)$, which completes the proof.

According to relation $(\mathcal{T})$ above, we obtain Lemma 2.7 as a corollary of Lemma 2.6.

Lemma 2.7. Let $f \in \mathcal{M}\left(d\left(a, R^{-}\right)\right)$. Then $T\left(r, f^{\prime}\right) \leqslant 2 T(r, f)+O(1)(r \in J)$. Moreover, if $f \in \mathcal{A}\left(d\left(a, R^{-}\right)\right)$, then $T\left(r, f^{\prime}\right) \leqslant T(r, f)+O(1)(r \in J)$. 
Lemma 2.8. Let $a, b, c, d \in K$ satisfy $a d-b c \neq 0$ and let $\phi, \psi \in \mathcal{A}\left(d\left(a, R^{-}\right)\right)$. There exists $A, B \in \mathbb{R}$ such that $\max (Z(r, \phi), Z(r, \psi))+A \geqslant \max (Z(r, a \phi+b \psi), Z(r, c \phi+d \psi)) \geqslant$ $\max (Z(r, \phi), Z(r, \psi))+B, \forall r \in J$.

Proof. Since $a, b$ cannot all equal zero, by Lemma 2.1 it is easily seen that

$$
Z(r, a \phi+b \psi) \leqslant \max (Z(r, \phi), Z(r, \psi))+O(1),
$$

and in the same way

$$
Z(r, c \phi+d \psi) \leqslant \max (Z(r, \phi), Z(r, \psi))+O(1) .
$$

Consequently, there exists a constant $A \in \mathbb{R}$ such that $\max (Z(r, a \phi+b \psi), Z(r, c \phi+d \psi)) \leqslant$ $\max (Z(r, \phi), Z(r, \psi))+B, \forall r \in J$. But since $a d-b c \neq 0$, putting $\theta=a \phi+b \psi, \tau=c \phi+d \psi$, we have $\phi=d \theta-b \tau, \psi=-c \theta+a \tau$, and therefore we get the constant $A$ such that the second inequality is satisfied.

Lemma 2.9. Let $\alpha_{1}, \ldots, \alpha_{q} \in K^{*}$ be pairwise distinct and let $\phi, \psi \in \mathcal{A}(K)$. There exists $A \in \mathbb{R}$ and for every $r \in J$ there exists $u(r) \in\{1, \ldots, q\}$ such that

$$
Z\left(r, \phi-\alpha_{j} \psi\right) \geqslant \max (Z(r, \phi), Z(r, \psi))+A \quad \forall j \neq u(r), 1 \leqslant j \leqslant q, \forall r \in J .
$$

Proof. Suppose Lemma 2.9 is not true. There exist two distinct indices $k, l \in\{1, \ldots$, $q\}$ and a sequence $\left(r_{n}\right)$ such that

$$
\begin{aligned}
& \lim _{n \rightarrow \infty}\left(\max \left(Z\left(r_{n}, \phi\right), Z\left(r_{n}, \psi\right)\right)-Z\left(r_{n}, \phi-\alpha_{k} \psi\right)\right)=+\infty, \\
& \lim _{n \rightarrow \infty}\left(\max \left(Z\left(r_{n}, \phi\right), Z\left(r_{n}, \psi\right)\right)-Z\left(r_{n}, \phi-\alpha_{l} \psi\right)\right)=+\infty .
\end{aligned}
$$

Thus, by Lemma 2.8 we are led to a contradiction.

Lemma 2.10 is classical and easily checked.

Lemma 2.10. Let $\alpha_{1}, \ldots, \alpha_{q} \in K$ be pairwise distinct and let $S=\left\{\alpha_{1}, \ldots, \alpha_{q}\right\}$. Let $f \in \mathcal{M}\left(d\left(0, R^{-}\right)\right)$. Then $\sum_{j=1}^{n}\left(Z\left(r, f-\alpha_{j}\right)-\bar{Z}\left(r, f-\alpha_{j}\right)\right)=Z\left(r, f^{\prime}\right)-Z_{0}^{S}\left(r, f^{\prime}\right) \forall r \in J$.

Proof of Theorems 1.4 and 1.4'. We can obviously assume that $f(0) \neq 0, f(0) \neq a_{i}$ $\forall i=1, \ldots, q, f(0) \neq \infty$. By Lemma 2.4 there exist $\phi, \psi \in \mathcal{A}\left(d\left(0, R^{-}\right)\right)$such that $f=\phi / \psi$, and

$$
\begin{aligned}
& Z(r, \phi) \leqslant Z(r, f)+1 \\
& Z(r, \psi) \leqslant N(r, f)+1
\end{aligned}
$$

By Lemma 2.9, there exists $A \in \mathbb{R}$ and for any $r \in J$, there exists $u(r) \in\{1, \ldots, q\}$ such that $Z\left(r, \phi-\alpha_{j} \psi\right) \geqslant \max (Z(r, \phi), Z(r, \psi))+A \forall j \neq u(r)$, therefore there exists $B \in \mathbb{R}$ such that

$$
Z\left(r, \phi-\alpha_{j} \psi\right) \geqslant T(r, f)+B \quad \forall j \neq u(r), \quad \forall r \in J .
$$


We check that $\mathcal{D}(\phi)-\mathcal{D}(\phi / \psi)=\mathcal{D}(\psi)-\mathcal{D}(\psi / \phi)$, therefore

$$
\mathcal{D}\left(\phi-\alpha_{j} \psi\right)=\mathcal{D}\left(f-\alpha_{j}\right)+\mathcal{D}(\psi)-\mathcal{D}\left(\frac{1}{f-\alpha_{j}}\right)=\mathcal{D}\left(f-\alpha_{j}\right)+\mathcal{D}(\psi)-\mathcal{D}\left(\frac{1}{f}\right) .
$$

Then, applying counting functions, we have $Z\left(r, \phi-\alpha_{j} \psi\right)=Z\left(r, f-\alpha_{j}\right)+Z(r, \psi)-$ $N(r, f)$, and therefore, by (2.3), we obtain

$$
Z\left(r, \phi-\alpha_{j} \psi\right) \leqslant Z\left(r, f-\alpha_{j}\right)+1 .
$$

Then, by (2.4) and (2.5) we obtain

$$
(q-1)(T(r, f)+B) \leqslant \sum_{\substack{1 \leqslant j \leqslant q, j \neq u(r)}} Z\left(r, \phi-\alpha_{j} \psi\right) \leqslant \sum_{\substack{1 \leqslant j \leqslant q, j \neq u(r)}} Z\left(r, f-\alpha_{j}\right)+q-1 \quad \forall r \in J .
$$

Putting $H=(q-1)(1-B)$, we obtain

$$
(q-1) T(r, f) \leqslant \sum_{j=1}^{q} Z\left(r, f-\alpha_{j}\right)+H-Z\left(r, f-\alpha_{u(r)}\right) \quad \forall r \in J .
$$

Now, by Lemma 2.10 we have

$$
\sum_{j=1}^{q} Z\left(r, f-\alpha_{j}\right)=\sum_{j=1}^{q} \bar{Z}\left(r, f-\alpha_{j}\right)+Z\left(r, f^{\prime}\right)-Z_{0}^{S}\left(r, f^{\prime}\right),
$$

hence by (2.6) we have

$$
(q-1) T(r, f) \leqslant \sum_{j=1}^{q} \bar{Z}\left(r, f-\alpha_{j}\right)+Z\left(r, f^{\prime}\right)-Z\left(r, f-\alpha_{u(r)}\right)-Z_{0}^{S}\left(r, f^{\prime}\right)+H \quad \forall r \in J .
$$

Suppose that $f$ belongs to $\mathcal{A}\left(d\left(a, R^{-}\right)\right)$. By Lemma 2.1 we have $Z\left(r, f-\alpha_{u(r)}\right)=T(r, f)+$ $O(1) \forall r \in J$, so, by (2.7), we obtain

$$
q T(r, f) \leqslant \sum_{j=1}^{q} \bar{Z}\left(r, f-\alpha_{j}\right)+Z\left(r, f^{\prime}\right)-Z_{0}^{S}\left(r, f^{\prime}\right)+H \quad \forall r \in J
$$

Returning to the general case, by Lemma 2.6 for every $j=1, \ldots, q$, we have a constant $c_{j} \in \mathbb{R}$ such that $Z\left(r, f^{\prime}\right) \leqslant Z\left(r, f-\alpha_{j}\right)+\bar{N}\left(r, f-\alpha_{j}\right)+c_{j} \forall r \in J$. Putting $M=$ $\max \left(c_{1}, \ldots, c_{q}\right)$, we have $Z\left(r, f^{\prime}\right) \leqslant Z\left(r, f-\alpha_{u(r)}\right)+\bar{N}\left(r, f-\alpha_{u(r)}\right)+M \forall r \in J$.

Moreover, $\bar{N}\left(r, f-\alpha_{u(r)}\right)=\bar{N}(r, f)$, hence

$$
Z\left(r, f^{\prime}\right) \leqslant Z\left(r, f-\alpha_{u(r)}\right)+\bar{N}(r, f)-\log r \quad \forall r \in J .
$$

Finally, by (2.7) and (2.8), we obtain

$$
(q-1) T(r, f) \leqslant \sum_{j=1}^{q} \bar{Z}\left(r, f-\alpha_{j}\right)+\bar{N}(r, f)-Z_{0}^{S}\left(r, f^{\prime}\right)+H+M-\log r \quad \forall r \in J .
$$

This completes the proof. 
Lemma 2.11 is easily checked.

Lemma 2.11. Let $\alpha_{1}, \ldots, \alpha_{n} \in K$ be pairwise distinct and let

$$
P(u)=\prod_{i=1}^{n}\left(u-\alpha_{i}\right),
$$

and let $f \in \mathcal{M}\left(d\left(0, R^{-}\right)\right)$. Then

$$
Z(r, P(f))=\sum_{i=1}^{n} Z\left(r, f-\alpha_{i}\right) \quad \text { and } \quad \bar{Z}(r, P(f))=\sum_{i=1}^{n} \bar{Z}\left(r, f-\alpha_{i}\right) .
$$

Lemma 2.12. Let $F \in \mathcal{M}\left(d\left(a, R^{-}\right)\right)$and let $L=F^{\prime} / F$. Then, $L$ satisfies $Z(r, L) \leqslant$ $N(r, L)+O(1)(r \in J)$.

Proof. Without loss of generality we can obviously assume that $L(0) L^{\prime}(0) \neq 0$. As a $\operatorname{logarithmic~derivative,~} L$ satisfies $v(L,-\log r) \geqslant \log r$ [5, Lemma 4]. On the other hand, by Lemma 2.1 we have $v(L,-\log r)=v(L(0))-Z(r, L)+N(r, L)$. Consequently, we obtain $v(L(0))-Z(r, L)+N(r, L) \geqslant \log r$, which proves the claim.

Lemma 2.13 is easily proven by classical considerations on limited developments [6].

Lemma 2.13. Let $F, G \in \mathcal{M}\left(d\left(a, R^{-}\right)\right)$have the same poles, ignoring multiplicities, and let $H=F^{\prime \prime} / F^{\prime}-G^{\prime \prime} / G^{\prime}$. Every pole of $H$ has multiplicity order 1 . Let $\alpha$ be a pole of $F$ and $G$. If $\alpha$ has same multiplicity for $F$ and $G$, then $H$ has no pole at $\alpha$. Moreover, if $\alpha$ has a multiplicity order 1 for both $F$ and $G$, then $\alpha$ is a zero of $H$.

Proof of Theorems 1.6 and 1.11. Suppose that $f \neq g$. In order to simplify the notation, we will denote by $P$ the polynomial $P_{n, c}$. Let $F=1 / P(f), G=1 / P(g)$ and $H=F^{\prime \prime} / F^{\prime}-G^{\prime \prime} / G^{\prime}$. Without loss of generality we may obviously assume that $a=0$, and that 0 is neither a zero nor a pole for $f, f^{\prime}, g, g^{\prime}$ and $H$.

We first suppose that $H$ is identically 0 . In this case, we will follow the same way as in [12]. Consequently, there exist $A \in K^{*}$ and $B \in K$ such that $P(f)=P(g) /(B P(g)+A)$. Therefore, by Theorem 1.2 we have

$$
T(r, f)=T(r, g)+O(1) \quad(r \in] 0, R[) .
$$

Since $f$ is unbounded, we deduce that $g$ is unbounded. As noticed in [12], $P(X)+c$ is of the form $D X^{n-2}\left(X-e_{1}\right)\left(X-e_{2}\right)$, with $D \in K, e_{1} e_{2} \neq 0, e_{1} \neq e_{2}$. We have to distinguish three cases: (i) $B \neq 0$, (ii) $B=0, A=1$, (iii) $B=0, A \neq 1$. Case (i) is dealt with as in [12]: we will only verify that the Nevanlinna formula applies without any difference. Cases (ii) and (iii) need to be thoroughly examined here.

(i) Since $A B \neq 0$, every zero of $P(g)+A / B$ is a pole of $P(f)$ and therefore is a zero of order at least $n$ of $P(g)+A / B$. On the other hand, we check that whenever $A, B \in K^{*}$, the polynomial $P(X)+A / B$ admits at least two distinct zeros $b_{1}$ and $b_{2}$ of order 1 and therefore it admits another zero $l$ of order at most $n-2$. Consequently, every zero of $g-b_{1}$ or $g-b_{2}$ has order at least $n$, and every zero of $g-l$ has order at least 2. By a 
change of variable, we can obviously assume that $g, g-l, g-b_{1}, g-b_{2}$ have neither any zeros nor any poles at 0 . Then, by applying Theorem 1.4, we obtain

$$
\begin{aligned}
2 T(r, g)-\bar{N}(r, g) & \leqslant \bar{Z}(r, g-l)+\bar{Z}\left(r, g-b_{1}\right)+\bar{Z}\left(r, g-b_{2}\right)+O(1) \\
& \leqslant \frac{1}{2} Z(r, g-l)+\frac{1}{n}\left(Z\left(r, g-b_{1}\right)+Z\left(r, g-b_{2}\right)\right)+O(1) \quad(r \in J),
\end{aligned}
$$

which leads to $n \leqslant 4$.

(ii) In this case we have $P(f)=P(g)$. Then putting $h=f / g$, we deduce that

$$
(n-1)(n-2)\left(h^{n}-1\right) g^{2}-2 n(n-2)\left(h^{n-1}-1\right) g+n(n-1)\left(h^{n-2}-1\right)=0 .
$$

If $h$ is a constant, it is 1 , a contradiction. So, we suppose $h$ not constant. Since $g$ lies in $\mathcal{M}_{u}\left(d\left(a, R^{-}\right)\right)$, it is easily seen that so does $h$. Indeed, suppose that $h \in \mathcal{M}_{b}\left(d\left(a, R^{-}\right)\right)$. Then clearly we have $T\left(r,(n-1)(n-2)\left(h^{n}-1\right) g^{2}\right) \geqslant 2 T(r, g)+O(1)$, while $T(r,-2 n(n-$ $\left.2)\left(h^{n-1}-1\right) g+n(n-1)\left(h^{n-2}-1\right)\right) \leqslant T(r, g)+O(1)$, a contradiction.

Now, let $\theta(X)=(n-1)^{2}\left(X^{n}-1\right)-n(n-2)\left(X^{n-1}-1\right)^{2}$. In [12] it is proven that, in $\mathbb{C}, \theta$ admits 1 as a zero of order 4 and that all other zeros $u_{j}(1 \leqslant j \leqslant 2 n-6)$ are simple. Of course, the proof applies to every algebraically closed field of characteristic zero. By a change of variable, we can obviously assume that $h-u_{j}$ has no zeros and no poles at 0 . Consequently, we check that

$$
\left(g-\left(\frac{n}{n-1}\right)\left(\frac{h^{n-1}-1}{h^{n}-1}\right)\right)^{2}=\frac{n(h-1)^{4} \prod_{j=1}^{2 n-6}\left(h-u_{j}\right)}{(n-1)^{2}(n-2)\left(h^{n}-1\right)^{2}} .
$$

Since

$$
\frac{n(h-1)^{4} \prod_{j=1}^{2 n-6}\left(h-u_{j}\right)}{(n-1)^{2}(n-2)\left(h^{n}-1\right)^{2}}
$$

is equal to a square, clearly each zero $h-u_{j}(1 \leqslant j \leqslant 2 n-6)$ has order at least 2 . Consequently,

$$
\sum_{j=1}^{2 n-6} \bar{Z}\left(r, h-u_{j}\right) \leqslant \frac{1}{2} \sum_{j=1}^{2 n-6} Z\left(r, h-u_{j}\right) \leqslant \frac{1}{2}(2 n-6) T(r, h)+O(1) \quad(r \in J) .
$$

Then, applying Theorem $1.4^{\prime}$ to $h$ at the points $u_{j}(1 \leqslant j \leqslant 2 n-6)$, we obtain

$$
\begin{aligned}
(2 n-7) T(r, h) & \leqslant \sum_{j=1}^{2 n-6} \bar{Z}\left(r, h-u_{j}\right)+\bar{N}(r, h)+O(1) \\
& \leqslant \frac{1}{2} \sum_{j=1}^{2 n-6} Z\left(r, h-u_{j}\right)+\bar{N}(r, h)+O(1) \\
& \leqslant \frac{1}{2}(2 n-6) T(r, h)+\bar{N}(r, h)+O(1) \quad(r \in J) .
\end{aligned}
$$

Since $T(r, h)$ is unbounded, we have $n \leqslant 5$. 
Now, suppose that both $f$ and $g$ lie in $\mathcal{A}_{1 / 5}\left(d\left(0, R^{-}\right)\right.$) (in particular, this happens when $f$ or $g$ belongs to $\mathcal{A}_{b d}\left(d\left(0, R^{-}\right)\right)$). Actually, we only have to consider the case when $n=5$. Let $V(X)=P_{5, c}(X)-c=2 X^{3}\left(6 X^{2}-15 X+10\right)$. Then $V(X)$ is of the form $2 X^{3}(X-a)(X-b)$ with $a \neq b$. Let $\alpha$ be a pole of $h$, hence a zero of $g$. Since $V(f)=V(g)$, either $\alpha$ is a zero of $f$, or it is a zero of $(f-a)(f-b)$. But if $\alpha$ is a zero of $f$, then we have $\omega_{\alpha}(f)=\omega_{\alpha}(g)$, therefore $\alpha$ is not a pole of $h$. Hence, we have $(f(\alpha)-a)(f(\alpha)-b)=0$, and

$$
\omega_{\alpha}(f-a)+\omega_{\alpha}(f-b)=\omega_{\alpha}(V(g))=3 \omega_{\alpha}(g) .
$$

Thus, each pole $\alpha$ of $h$ is a zero of order at least 3 either for $f-a$ or for $f-b$. Consequently, we have $2 \bar{N}(r, h) \leqslant Z(r, f-a)-\bar{Z}(r, f-a)+Z(r, f-b)-\bar{Z}(r, f-b)$. And since $Z(r, f-a)-\bar{Z}(r, f-a)+Z(r, f-b)-\bar{Z}(r, f-b) \leqslant Z\left(r, f^{\prime}\right)$, we have $2 \bar{N}(r, h) \leqslant Z\left(r, f^{\prime}\right)$. But since $f \in \mathcal{A}_{1 / 5}\left(d\left(0, R^{-}\right)\right)$, we obtain

$$
\limsup _{r \rightarrow R} \frac{\bar{N}(r, h)}{T(r, f)}<\frac{1}{10}
$$

On the other hand, by $(2.11)$ we check that $\omega_{\alpha}\left(f^{\prime}\right)=3 \omega_{\alpha}(g)-1=-3 \omega_{\alpha}(h)-1$, hence $3 N(r, h)-\bar{N}(r, h) \leqslant Z\left(r, f^{\prime}\right)$. Consequently,

$$
\limsup _{r \rightarrow R} \frac{N(r, h)}{T(r, f)} \leqslant \frac{1}{3} \limsup _{r \rightarrow R}\left(\frac{\bar{N}(r, h)}{T(r, f)}+\frac{Z\left(r, f^{\prime}\right)}{T(r, f)}\right) .
$$

Now, since $f \in \mathcal{A}_{1 / 5}\left(d\left(0, R^{-}\right)\right)$, by (2.12) we obtain

$$
\limsup _{r \rightarrow R} \frac{N(r, h)}{T(r, f)}<\frac{1}{10} .
$$

By Lemma 2.4 we can find $\phi, \psi \in \mathcal{A}\left(d\left(0, R^{-}\right)\right)$such that $h=\phi / \psi, \phi(0) \psi(0) \neq 0$, and $Z(r, \phi) \leqslant Z(r, h)+1, Z(r, \psi) \leqslant N(r, h)+1$. Since $T(r, f)=T(r, g)+O(1)(r \in] 0, R[)$ when $r$ approaches $R$, it is seen that $|Z(r, f)-Z(r, g)|_{\infty}$ is bounded, and by Lemma 2.1 , so is $v(h, \mu)$ when $\mu$ approaches $-\log R$. Consequently, $|Z(r, \phi)-Z(r, \psi)|_{\infty}$ is also bounded when $r$ approaches $R$. Therefore, by (2.13) we have

$$
\limsup _{r \rightarrow R} \frac{T(r, h)}{T(r, g)}<\frac{1}{10}
$$

Consequently, by Lemma 2.1, we have

$$
\begin{aligned}
& \limsup _{\mu \rightarrow-\log R} \frac{v(\phi, \mu)}{v(g, \mu)}<\frac{1}{10}, \\
& \limsup _{\mu \rightarrow-\log R} \frac{v(\psi, \mu)}{v(g, \mu)}<\frac{1}{10} .
\end{aligned}
$$

Now, by (2.10), we check that

$$
\left[g\left(\phi^{5}-\psi^{5}\right)-\left(\frac{5}{4}\right)\left(\phi^{4} \psi-\psi^{5}\right)\right]^{2}=\frac{5(\psi-\phi)^{4} \prod_{j=1}^{4}\left(\phi-u_{j} \psi\right)}{48 \psi^{3}} .
$$


Now, by (2.9), (2.14) and (2.15), it is easily seen that

$$
\begin{aligned}
\lim _{\mu \rightarrow-\log R}(v(g, \mu)-4 v(\phi, \mu)-v(\psi, \mu)) & =-\infty, \\
\lim _{\mu \rightarrow-\log R}(v(g, \mu)-5 v(\phi, \mu)) & =-\infty,
\end{aligned}
$$

and therefore, when $\mu$ approaches $-\log R$, we have $v\left(g\left(\phi^{5}-\psi^{5}\right), \mu\right)<v\left(\frac{5}{4}\left(\phi^{4}-\psi^{4}\right) \psi, \mu\right)$. Hence

$$
v\left(\left[g\left(\phi^{5}-\psi^{5}\right)-\left(\frac{5}{4}\right)\left(\phi^{4} \psi-\psi^{5}\right)\right]^{2}, \mu\right)=v\left(g\left(\phi^{5}-\psi^{5}\right), \mu\right) \leqslant v(g, \mu) .
$$

On the other hand, we can check that

$$
v\left(\frac{5(\psi-\phi)^{4} \prod_{j=1}^{4}\left(\phi-u_{j} \psi\right)}{48 \psi^{3}}, \mu\right) \geqslant 5 v(\phi, \mu)+O(1) \quad(\mu \in]-\log R,+\infty[) .
$$

Then by (2.9), (2.14) and (2.15) we obtain

$$
v\left(\frac{5(\psi-\phi)^{4} \prod_{j=1}^{4}\left(\phi-u_{j} \psi\right)}{48 \psi^{3}}, \mu\right) \geqslant \frac{5}{4} v(g, \mu)+O(1) \quad(\mu \in]-\log R,+\infty[)
$$

a contradiction to (2.16) and (2.17). This shows that the hypothesis ' $h$ non-constant' is absurd. This finishes proving that $n \leqslant 4$ when $f \in \mathcal{A}_{1 / 5}\left(d\left(0, R^{-}\right)\right)$, and $g \in$ $\mathcal{A}_{1 / 5}\left(d\left(0, R^{-}\right)\right) \cup \mathcal{A}_{b d}\left(d\left(0, R^{-}\right)\right)$in case (ii).

(iii) Let $\lambda=1 / A$. We will check that at least one of the two polynomials

$$
Q_{1}(X)=(n-1)(n-2) X^{n}-2 n(n-2) X^{n-1}+n(n-1) X^{n-2}+c(\lambda-1)
$$

and

$$
Q_{2}(X)=(n-1)(n-2) X^{n}-2 n(n-2) X^{n-1}+n(n-1) X^{n-2}+c\left(\frac{1}{\lambda}-1\right)
$$

admits $n$ distinct zeros. Indeed, suppose this not true. We notice that

$$
Q_{1}^{\prime}(X)=Q_{2}^{\prime}(X)=P^{\prime}(X)=n(n-1)(n-2) X^{n-3}(X-1)^{2} .
$$

Consequently, if both $Q_{1}$ and $Q_{2}$ admits at least one zero of order $>1$, then this must be 1 , because it cannot be 0 . But then, we have $c(\lambda-1)=c(1 / \lambda-1)=-2$. Since $c \neq 0$, and since $\lambda \neq 1$, this implies $\lambda=-1$, and $c=1$, which is excluded by hypothesis.

Now, since $\lambda$ and $1 / \lambda$ play symmetric roles, without loss of generality we can assume that $Q_{1}$ admits $n$ distinct zeros $b_{1}, \ldots, b_{n}$. Thus, putting

$$
\Gamma(X)=\lambda X^{n-2}(n-1)\left((n-2) X^{2}-2 n(n-2) X+n(n-1)\right),
$$

we have

$$
Q_{1}(f)=\Gamma(g)
$$


So, applying Theorem $1.4^{\prime}$, we obtain

$$
(n-1) T(r, f) \leqslant \sum_{j=1}^{n} \bar{Z}\left(r, f-b_{j}\right)+\bar{N}(r, f)+O(1) \quad(r \in J) .
$$

On the other hand, by Lemma 2.11 we have

$$
\sum_{j=1}^{n} \bar{Z}\left(r, f-b_{j}\right) \leqslant \bar{Z}\left(r, Q_{1}(f)\right)=\bar{Z}(r, g)+\bar{Z}\left(r, g-e_{1}\right)+\bar{Z}\left(r, g-e_{2}\right) \leqslant 3 T(r, g) .
$$

But by $(2.18)$ it is seen that $T(r, g) \leqslant T(r, f)+O(1)(r \in J)$, hence finally

$$
(n-1) T(r, f) \leqslant 3 T(r, f)+\bar{N}(r, f)+O(1) \quad(r \in J) .
$$

Consequently, we have $n \leqslant 5$. Moreover, if $f, g \in \mathcal{A}\left(d\left(0, R^{-}\right)\right)$, then $N(r, f)=0$, and then (2.19) leads to $n \leqslant 4$. This finishes proving the claims of Theorems 1.6 and 1.11 when $H=0$.

Henceforth, we suppose that $H$ is not identically 0 and will apply Fujimoto's method. Let $\alpha$ be a zero of $f-a_{j}$, for some $j$. Then both $P(f)$ and $P(g)$ vanish at $\alpha$. So, by Lemma 2.11 we have $\sum_{j=1}^{n} \bar{Z}\left(r, f-a_{j}\right)=\bar{Z}(r, P(f))=\bar{Z}(r, P(g))$. Now, according to Lemma 2.13, if both $P(f), P(g)$ have a zero of order one, then $H$ also has a zero. Else, at least one of the two functions $P(f)$ and $P(g)$ admits $\alpha$ as a zero of order strictly greater than 1 , and then (since all zeros of $P$ are simple) at least one of the two functions $f^{\prime}, g^{\prime}$ has a zero at $\alpha$, while $\alpha$ is a zero $f-a_{i}$ for some $i$ and a zero of $g-a_{j}$ for some $j$. Consequently, we obtain

$$
\sum_{j=1}^{n} \bar{Z}\left(r, f-a_{j}\right) \leqslant \bar{Z}(r, H)+\bar{Z}\left(r, f^{\prime}\right)+\bar{Z}\left(r, g^{\prime}\right)-\bar{Z}_{0}^{S}\left(r, f^{\prime}\right)-\bar{Z}_{0}^{S}\left(r, g^{\prime}\right) .
$$

Moreover, in the hypothesis of Theorem 1.11, since any zero $\alpha$ of $f-a_{i}$ is a zero of certain $g-a_{j}$ with the same multiplicity order we have $\omega_{\alpha}\left(f^{\prime}\right)=\omega_{\alpha}\left(g^{\prime}\right)$. Consequently, we obtain this improvement of $(2.20 a)$ :

$$
\sum_{j=1}^{n} \bar{Z}\left(r, f-a_{j}\right) \leqslant \bar{Z}(r, H)+\frac{1}{2}\left[\bar{Z}\left(r, f^{\prime}\right)+\bar{Z}\left(r, g^{\prime}\right)-\bar{Z}_{0}^{S}\left(r, f^{\prime}\right)-\bar{Z}_{0}^{S}\left(r, g^{\prime}\right)\right] .
$$

By Lemma 2.12 we know that $\bar{Z}(r, H) \leqslant N(r, H)+O(1)(r \in J)$, hence

$\sum_{j=1}^{n} \bar{Z}\left(r, f-a_{j}\right) \leqslant N(r, H)+\bar{Z}\left(r, f^{\prime}\right)+\bar{Z}\left(r, g^{\prime}\right)-\bar{Z}_{0}^{S}\left(r, f^{\prime}\right)-\bar{Z}_{0}^{S}\left(r, g^{\prime}\right)+O(1) \quad(r \in J)$,

and similarly

$$
\sum_{j=1}^{n} \bar{Z}\left(r, g-a_{j}\right) \leqslant N(r, H)+\bar{Z}\left(r, f^{\prime}\right)+\bar{Z}\left(r, g^{\prime}\right)-\bar{Z}_{0}^{S}\left(r, f^{\prime}\right)-\bar{Z}_{0}^{S}\left(r, g^{\prime}\right)+O(1) \quad(r \in J),
$$


hence

$\sum_{j=1}^{n} \bar{Z}\left(r, f-a_{j}\right)+\bar{Z}\left(r, g-a_{j}\right) \leqslant 2 N(r, H)+2\left[\bar{Z}\left(r, f^{\prime}\right)+\bar{Z}\left(r, g^{\prime}\right)-\bar{Z}_{0}^{S}\left(r, f^{\prime}\right)-\bar{Z}_{0}^{S}\left(r, g^{\prime}\right)\right]$.

And in the hypothesis of Theorem 1.11, by $(2.20 \mathrm{~b})$ we obtain

$$
\sum_{j=1}^{n} \bar{Z}\left(r, f-a_{j}\right)+\bar{Z}\left(r, g-a_{j}\right) \leqslant 2 N(r, H)+\left[\bar{Z}\left(r, f^{\prime}\right)+\bar{Z}\left(r, g^{\prime}\right)-\bar{Z}_{0}^{S}\left(r, f^{\prime}\right)-\bar{Z}_{0}^{S}\left(r, g^{\prime}\right)\right] .
$$

Consider now $N(r, H)$, and let $\beta$ be a pole of $H$. Either $\beta$ is a zero of $F^{\prime} G^{\prime}$ or it is a pole of $F G$. Let $\psi$ be the counting function of the poles of $H$ occurring when $F G$ has a pole. So, we have

$$
N(r, H)=\bar{Z}\left(r, F^{\prime} G^{\prime}\right)+\varphi(r) .
$$

Suppose first $\beta$ is a zero of $F^{\prime}$ but is not a pole of $F G$. Either $\beta$ is a zero of $f^{\prime}$, or it is a zero of $P^{\prime}(f)$, or it is a pole of $f$. Consequently, $\beta$ is not a zero of $P(f)$ and we have $\bar{Z}\left(r, F^{\prime}\right) \leqslant \bar{Z}_{0}^{S}\left(r, f^{\prime}\right)+\bar{Z}\left(r, P^{\prime}(f)\right)+\bar{N}(r, f)$. Since

$$
P^{\prime}(X)=n(n-1)(n-2)(X-1)^{2} X^{n-3},
$$

we have $\bar{Z}\left(r, P^{\prime}(f)\right) \leqslant 2 T(r, f), \bar{Z}\left(r, P^{\prime}(g)\right) \leqslant 2 T(r, g)$, and therefore

$$
\bar{Z}\left(r, F^{\prime}\right) \leqslant \bar{Z}_{0}^{S}\left(r, f^{\prime}\right)+2 T(r, f)+\bar{N}(r, f) .
$$

Similarly, if $\beta$ is a zero of $G^{\prime}$ but is not a pole of $F G$, we have

$$
\bar{Z}\left(r, G^{\prime}\right) \leqslant \bar{Z}_{0}^{S}\left(r, g^{\prime}\right)+2 T(r, g)+\bar{N}(r, g) .
$$

Suppose now $\beta$ is a pole of $F G$. Then $\beta$ is a zero of $P(f)$ and $P(g)$ (we notice that when $\beta$ is a zero of $P(f)$, it is a zero of $P(g)$, and vice versa), and then by Lemma 2.13, it cannot be a pole of $H$ when it is a zero of same order of $P(f)$ and $P(g)$. Consequently, in the hypothesis of Theorem 1.6, $\varphi(r)$ satisfies

$$
\varphi(r) \leqslant \bar{Z}\left(r, f^{\prime}\right)-\bar{Z}_{0}^{S}\left(r, f^{\prime}\right)+\bar{Z}\left(r, g^{\prime}\right)-\bar{Z}_{0}^{S}\left(r, g^{\prime}\right),
$$

and therefore by $(2.22),(2.23)$ and $(2.24)$ we obtain

$$
\begin{aligned}
N(r, H) \leqslant \bar{Z}_{0}^{S}\left(r, f^{\prime}\right)+\bar{Z}_{0}^{S}\left(r, g^{\prime}\right)+ & 2 T(r, f)+2 T(r, g)+\bar{N}(r, f)+\bar{N}(r, g) \\
& +\bar{Z}\left(r, f^{\prime}\right)-\bar{Z}_{0}^{S}\left(r, f^{\prime}\right)+\bar{Z}\left(r, g^{\prime}\right)-\bar{Z}_{0}^{S}\left(r, g^{\prime}\right),
\end{aligned}
$$

hence

$$
N(r, H) \leqslant 2 T(r, f)+2 T(r, g)+\bar{N}(r, f)+\bar{N}(r, g)+\bar{Z}\left(r, f^{\prime}\right)+\bar{Z}\left(r, g^{\prime}\right) .
$$


Thus by $(2.21 a)$, in the hypothesis of Theorem 1.6, we obtain

$$
\begin{aligned}
\sum_{j=1}^{n} \bar{Z}\left(r, f-a_{j}\right)+ & \bar{Z}\left(r, g-a_{j}\right) \\
\leqslant & 4(T(r, f)+T(r, g))+2(\bar{N}(r, f)+2 \bar{N}(r, g)) \\
& +4\left(\bar{Z}\left(r, f^{\prime}\right)+\bar{Z}\left(r, g^{\prime}\right)\right)+O(1)
\end{aligned}
$$

Now, in the hypotheses of Theorem 1.11, since the order of a zero is the same for $P(f)$ and $P(g)$, the counting function $\psi$ is identically 0 , so, by $(2.22)-(2.24)$, we have

$$
N(r, H) \leqslant 2 T(r, f)+2 T(r, g)+\bar{N}(r, f)+\bar{N}(r, g)+\bar{Z}_{0}^{S}\left(r, f^{\prime}\right)+\bar{Z}_{0}^{S}\left(r, g^{\prime}\right),
$$

and therefore by $(2.21 b)$ we obtain

$$
\begin{aligned}
& \sum_{j=1}^{n} \bar{Z}\left(r, f-a_{j}\right)+\bar{Z}\left(r, g-a_{j}\right) \\
& \leqslant 4(T(r, f)+T(r, g))+2(\bar{N}(r, f)+2 \bar{N}(r, g))+\left(\bar{Z}\left(r, f^{\prime}\right)\right. \\
&\left.+\bar{Z}\left(r, g^{\prime}\right)\right)+\left(\bar{Z}_{0}^{S}\left(r, f^{\prime}\right)+\bar{Z}_{0}^{S}\left(r, g^{\prime}\right)\right)+O(1) .
\end{aligned}
$$

Now, applying Theorem $1.4^{\prime}$ to $f$ and $g$ at the points $a_{j}(1 \leqslant j \leqslant n)$, we have

$$
\begin{array}{ll}
(n-1) T(r, f) \leqslant \bar{N}(r, f)+\sum_{j=1}^{n} \bar{Z}\left(r, f-a_{j}\right)-Z_{0}^{S}\left(r, f^{\prime}\right)+O(1) & (r \in J), \\
(n-1) T(r, g) \leqslant \bar{N}(r, g)+\sum_{j=1}^{n} \bar{Z}\left(r, g-a_{j}\right)-Z_{0}^{S}\left(r, g^{\prime}\right)+O(1) & (r \in J),
\end{array}
$$

hence

$$
\begin{aligned}
& (n-1)(T(r, f)+T(r, g)) \\
& \leqslant \bar{N}(r, f)+\bar{N}(r, g)+\sum_{j=1}^{n} \bar{Z}\left(r, f-a_{j}\right) \\
& \quad+\bar{Z}\left(r, g-a_{j}\right)-Z_{0}^{S}\left(r, f^{\prime}\right)-Z_{0}^{S}\left(r, g^{\prime}\right)+O(1) \quad(r \in J),
\end{aligned}
$$

hence by $(2.25 a)$, in the hypotheses of Theorem 1.6, we obtain

$$
\begin{aligned}
& (n-1)(T(r, f)+T(r, g)) \\
& \leqslant 3(\bar{N}(r, f)+\bar{N}(r, g))+4(T(r, f)+T(r, g)) \\
& \quad+4\left(\bar{Z}\left(r, f^{\prime}\right)+\bar{Z}\left(r, g^{\prime}\right)\right)+O(1) \quad(r \in J) .
\end{aligned}
$$

And by $(2.25 b)$, in the hypotheses of Theorem 1.11, we obtain

$$
\begin{aligned}
& (n-1)(T(r, f)+T(r, g)) \\
& \leqslant 3(\bar{N}(r, f)+\bar{N}(r, g))+4(T(r, f)+T(r, g)) \\
& +\bar{Z}\left(r, f^{\prime}\right)+\bar{Z}\left(r, g^{\prime}\right)+O(1) \quad(r \in J) .
\end{aligned}
$$


Suppose that we assume the hypotheses of Theorem 1.6. Then, since $T(r, f)+T(r, g)$ is unbounded, we obtain $n \leqslant 16$. This shows the first claim. We now suppose that $f$ and $g$ belong to $\mathcal{A}_{u}\left(d\left(0, R^{-}\right)\right)$. On the one hand $\bar{N}(r, f)+\bar{N}(r, g)=0$. On the other hand, by Lemma 2.7 we have $T\left(r, f^{\prime}\right) \leqslant T(r, f)-\log r$, hence by $(2.26 a)$, we obtain $n \leqslant 9$. This shows the second claim.

We now assume the hypotheses of Theorem 1.11. By Lemma 2.7 we have $Z\left(r, f^{\prime}\right) \leqslant$ $2 T(r, f)+O(1)$, so, by Relation $(2.26 b)$ we obtain $n \leqslant 10$, which proves the first claim. Next, we suppose that $f$ and $g$ belong to $\mathcal{A}_{u}\left(d\left(a, r^{-}\right)\right)$. Then $\bar{N}(r, f)+\bar{N}(r, g)=0$ and by Lemma 2.7 we have $T\left(r, f^{\prime}\right) \leqslant T(r, f)-\log r$, hence by $(2.26 b)$, we obtain $n \leqslant 6$. This ends the proof of Theorem 1.11.

Thus it only remains for us to prove the claims of Theorem 1.6 when $f, g$ belong to $\mathcal{M}_{1 / 5}\left(d\left(a, R^{-}\right)\right)$, and when $f, g$ belong to $\mathcal{A}_{1 / 5}\left(d\left(a, R^{-}\right)\right)$, or $f$ belongs to $\mathcal{A}_{1 / 5}\left(d\left(a, R^{-}\right)\right)$ while $g$ belongs to $\mathcal{A}_{b d}\left(d\left(a, R^{-}\right)\right)$.

First, suppose that $f, g$ belongs to $\mathcal{M}_{1 / 5}\left(d\left(a, R^{-}\right)\right)$. By applying Theorem 1.4 instead of Theorem $1.4^{\prime}$, we obtain

$$
\begin{array}{ll}
(n-1) T(r, f) \leqslant \sum_{j=1}^{n} \bar{Z}\left(r, f-a_{j}\right)+Z\left(r, f^{\prime}\right)-Z_{0}^{S}\left(r, f^{\prime}\right)+O(1) & (r \in J), \\
(n-1) T(r, g) \leqslant \sum_{j=1}^{n} \bar{Z}\left(r, g-a_{j}\right)+Z\left(r, g^{\prime}\right)-Z_{0}^{S}\left(r, g^{\prime}\right)+O(1) & (r \in J) .
\end{array}
$$

Hence, by $(2.25 a)$,

$$
\begin{aligned}
(n-1)[T(r, f)+T(r, g)] \leqslant 4 & (T(r, f)+T(r, g))+2(\bar{N}(r, f)+\bar{N}(r, g)) \\
& +4\left(\bar{Z}\left(r, f^{\prime}\right)+\bar{Z}\left(r, g^{\prime}\right)\right)+\bar{Z}\left(r, f^{\prime}\right) \\
& -Z_{0}^{S}\left(r, f^{\prime}\right)+\bar{Z}\left(r, g^{\prime}\right)-Z_{0}^{S}\left(r, g^{\prime}\right)+O(1) \quad(r \in J) .
\end{aligned}
$$

Since $f, g$ lie in $\mathcal{M}_{1 / 5}\left(d\left(0, R^{-}\right)\right)$, it is clearly seen that

$$
\lim _{r \rightarrow R}\left(T(r, f)+T(r, g)-5\left(T\left(r, f^{\prime}\right)+T\left(r, g^{\prime}\right)\right)\right)=+\infty .
$$

Consequently, $n-1<4+2+5\left(\frac{1}{5}\right)$, i.e. $n \leqslant 7$.

Finally, suppose that $f$ and $g$ belong to $\mathcal{A}_{1 / 5}\left(d\left(a, r^{-}\right)\right)$, or that $f \in \mathcal{A}_{1 / 5}\left(d\left(a, r^{-}\right)\right)$and $g \in \mathcal{A}_{b d}\left(d\left(a, r^{-}\right)\right)$. In both cases, we can check

$$
\lim _{r \rightarrow R}\left(T(r, f)+T(r, g)-5\left(T\left(r, f^{\prime}\right)+T\left(r, g^{\prime}\right)\right)\right)=+\infty .
$$

Now, by Theorem 1.4 we obtain

$$
\begin{aligned}
& n(T(r, f)+t(r, g)) \\
& \quad \leqslant \sum_{j=1}^{n}(\bar{Z}(r, f-a-j)+\bar{Z}(r, g-a-j))+\bar{Z}\left(r, f^{\prime}\right)+\bar{Z}\left(r, g^{\prime}\right)+O(1),
\end{aligned}
$$


hence by $(2.25 a)$ we have

$$
n(T(r, f)+T(r, g)) \leqslant 4(T(r, f)+T(r, g))+5\left(\bar{Z}\left(r, f^{\prime}\right)+Z\left(r, g^{\prime}\right)\right)+O(1),
$$

so by $(2.27)$ we obtain $n \leqslant 4$, and this ends the proof of Theorem 1.6.

Remark. An interesting virtue of Fujimoto's method towards $\mathcal{A}_{1 / 5}\left(d\left(a, R^{-}\right)\right)$and $\mathcal{M}_{1 / 5}\left(d\left(a, R^{-}\right)\right)$, is that $(2.25 a)$ and $(2.25 b)$ are equivalent for functions $f$ and $g$ in these sets. Consequently, in these particular sets, looking for urs or for ursim through this method becomes equivalent.

Acknowledgements. We thank the referee for suggesting that we extend our survey to meromorphic functions.

\section{References}

1. W. W. Adams and E. G. Straus, Non archimedian analytic functions taking the same values at the same points, Illinois J. Math. 15 (1971), 418-424.

2. A. BoutabaA, Théorie de Nevanlinna p-adique, Manuscr. Math. 67 (1990), 251-269.

3. A. BoutabaA, Applications de la théorie de Nevanlinna $p$-adique, Collectanea Math. 42(1) (1991), 75-93.

4. A. Boutabaa, A. Escassut And L. Haddad, On uniqueness of $p$-adic entire functions, Indagationes Math. 8 (1997), 145-155.

5. A. Boutabaa and A. Escassut, On uniqueness of $p$-adic meromorphic functions, Proc. Am. Math. Soc. 126 (1998), 2557-2568.

6. A. Boutabaa and A. Escassut, Property $f^{-1}(S)=g^{-1}(S)$ for $p$-adic entire and meromorphic functions, Rendi. Circ. Mat. Palermo Ser. II 49 (2000), 501-520.

7. A. Boutaban AND A. Escassut, An improvement of the p-adic Nevanlinna theory and application to meromorphic functions, Lecture Notes in Pure and Applied Mathematics, no. 207 (Marcel Dekker, 1999).

8. W. CherRy AND Ye. ZhUAn, Non-archimedean Nevanlinna Theory in several variables and the non-archimedean Nevanlinna inverse problem, Trans. Am. Math. Soc. 349 (1997), 5043-5071.

9. W. CherRy AND C. C. YANG, Uniqueness of non-archimedean entire functions sharing sets of values counting multiplicities, Proc. Am. Math. Soc. 127 (1998), 967-971.

10. A. EsCASSUt, Analytic elements in p-adic analysis (Singapore, World Scientific, 1995).

11. A. Escassut, L. HAdDad And R. Vidal, Urs, ursim and non-urs for $p$-adic functions and polynomials, J. Number Theory $\mathbf{7 5}$ (1999), 133-144.

12. G. FRANK AND M. REINDERS, A unique range set for meromorphic functions with 11 elements, Complex Variable Theory Applic. 37 (1998), 185-193.

13. H. Fujimoto, On uniqueness of meromorphic functions sharing finite sets, Am. J. Math. 122 (2000), 1175-1203.

14. F. Gross And C. C. YAng, On preimage and range sets of meromorphic functions, Proc. Japan. Acad. 58(1) (1982), 17.

15. H. H. KhoÁI And M. V. QuAng, On p-adic Nevanlinna theory, Lecture Note in Mathematics, no. 1351, pp. 146-158 (Springer, 1988).

16. M. LAZARD, Les zéros des fonctions analytiques sur un corps valué complet, IHES Publ. Math. 14 (1962), 47-75.

17. P. Li And C. C. YAng, On the unique range set of meromorphic functions, Proc. Am. Math. Soc. 124 (1996), 177-185. 
18. P. Li AND C. C. YANG, Some further results on the unique range sets of meromorphic functions, Kodai Math. J. 18 (1995), 437-450.

19. E. Mues And M. REInders, Meromorphic functions sharing one value and unique range sets, Kodai Math. J. 18 (1995), 515-522.

20. I. V. Ostrovskit, F. B. Pakovitch And M. G. Zaidenberg, A remark on complex polynomials of least deviation, Int. Math. Res. Notices 14 (1996), 699-703.

21. P. C. Hu AND C. C. YAnG, A unique range set of $p$-adic functions meromorphic functions with 10 elements, Acta Math. Vietnamica 24(1) (1999), 95-108.

22. P. C. HU AND C. C. YANG, Meromorphic functions over non archimedean fields (Kluwer, 2000). 